\title{
Study on Campus Football Talent Training Mode for Primary and Middle Schools in Yanbian
}

\author{
Longhua Li ${ }^{1}$, Yijun Wang ${ }^{2, *}$ \\ ${ }^{1,2}$ Yanbian University, Yanji, Jilin, 133002, China \\ *Corresponding author
}

Keywords: Yanbian, Football in primary and middle schools, Top talents, training mode.

\begin{abstract}
Enhancing training of campus top football talents in primary and middle schools can effectively drive football career development of China, improve football sports level, make China's football career not fall behind others in the world and better establish the image of strong country. Selecting and training top football players in primary and middle schools is a main way to train top football players and bench players. Choosing football players from primary and middle school students can better achieve the purpose of training from childhood. Physical quality of primary and middle school students is in the good stage, with strong plasticity. Besides, they have strong learning ability for theoretical knowledge and also own excellent practical ability.
\end{abstract}

\section{Introduction}

This period is the best period for choosing and training talents. Currently, football has been fashionable in the world. People's attention to World Cup continues to rise, and only Olympic Games can be compared with it. In such situation, how to discover football talents in primary and middle schools and inject fresh blood for China's football career has become a social issue which has received extensive attention. This paper will analyze and study top talent training mode for campus football of primary and middle schools in Yanbian.

\section{Recent achievements of campus football in Yanbian}

\subsection{Established campus football association and promoted football career development}

Yanbian has been concerned about improvement and development of campus football of primary and middle schools, and it is also one of key job contents of sports department. On April 28, 2017, the first member congress of Campus Football Association of Yanbian Korean Autonomous Prefecture was held in Yanji. In the conference, Articles of Campus Football Association of Yanbian Korean Autonomous Prefecture were passed, and main posts were elected such as members, president, vice president, secretary-general and deputy secretary-general. Campus Football Association of Yanbian Korean Autonomous Prefecture is a mass social group which consists of Education Bureau, Sports Bureau, College of Education, Yanbian University and campus football workers. The association aims to better drive campus football development in Yanbian, enhance academic exchange among football talents in each field, cooperation and friendship, construct a more perfect communication channel between the government and mass, promote development and popularization of campus football, carry forward football culture, create favorable campus football atmosphere and establish scientific and systematic football top talent training mode for primary and middle schools, train professional football talents on campus, provide more excellent backup football players and further drive rapid development of teenager football career in China.

\subsection{Actively held campus league football matches in primary and middle schools}

Both the quality and times of campus league football matches for primary and middle school 
students in Yanbian have been in the leading position in China. Football has a long history in Yanbian. There are numerous football matches. The participants of campus league football matches for primary and middle schools are mainly primary and middle school students in each area. The groups are classified strictly, and the competition process is regular. Little football players own favorable techniques, and football competition has strong ornamental value. Meanwhile, the favorable development of primary and middle school football in Yanbian can be obviously seen.

\section{Effective training mode of campus top football talents in primary and middle schools of Yanbian}

\subsection{To pay attention to choosing top talents in primary and middle schools}

To make football team show new vitality and reduce work pressure of trainers, it is required to improve training efficiency, mainly cultivate the talents who have football talent or perform outstandingly in ordinary training and promote the whole level of football team. For any kind of sports, talent plays a great role. Each individual of primary and middle school students owns different talent and ability, not all students are suitable for football training. To become an excellent football player, strong physical strength, body coordination ability and agility are required. More importantly, favorable sense of foot, i.e. dribbling ability and shooting ability should be owned. Although these abilities can be promoted in effective trainings, the students with favorable foot talent have strong training acceptance ability and learning ability, and can well grasp football rules and features. For example, for the item which requires excellent footwork such as fixed-point shooting, even if some people have received long-term trainings, they cannot reach the level of professional football athletes. This indicates the importance of selecting players with talent from primary and middle schools. During picking out players, instructors must be careful and responsible, and deeply investigate football level of every student to avoid omission of some students with football talent and talent waste. Only with careful and responsible attitude, excellent football players can be better chosen, which contributes to cultivation of excellent teenager talents. In the selection process, physical characteristics and psychological characteristics of students in the age group should be combined to avoid poor performance due to tension. During football player selection in middle schools, physical development, physical strength, tactics idea, technical level and psychological quality of students should be tested.

\subsection{Selection mode of campus football players in primary and middle schools}

Generally, there are five selection requirements. Firstly, they should own the fastest speed among the peers, strong explosive power, favorable body coordination ability and very strong endurance in running sports. Secondly, they should own favorable psychological quality, optimistic and self-confident living state, and strong anti-pressure capacity, and will not give up easily. Thirdly, they should have good accuracy, and be able to keep favorable accuracy in the fast motion, accurately cognize the running position and judge accurate falling point of football, make correct judgments according to teammates' position and mobile position and pass the ball correctly. In other words, favorable consciousness is required in football games. Fourthly, they should possess excellent comprehension, fast understand and grasp football skills proposed by the coach, give play to these skills rationally and flexibly in football games. In the face of any problem in the competition, they can eliminate it and make correct judgments and actions. Fifthly, excellent football players should own very strong teamwork spirit. Football belongs to a team sports. In the competition process, the final victory can be gained only through favorable teamwork cooperation. Therefore, players should blend in the team, get along well with every teammate and respect every member.

\subsection{To innovate football teaching mode and organically combine football and teaching}

At present, educational level of athletes is poor generally, which is related to traditional athlete training mode. This to certain degree hinders sound development of football and brings about the 
adverse effect to the development of youth athletes. Football needs to consume lots of time for training, so effective learning time will be reduced. Just because of this contradiction, some parents do not hope their children engage in sports. If no good performance is achieved in sports, they cannot find the good way for living after entering the society due to no favorable knowledge reserve. Nonsupport of parents is also one of important reasons for slow development of football development in China. To solve this problem, teaching mode and football training mode should innovate. Students' integrated development can be promoted through enhancing training of students' physical exercise in daily teaching. Besides, it is required to attach importance to cultivating students' culture courses in some characteristic football schools so as to better improve quality of football athletes. Professional football requires plenty of time for training. Especially, lots of time and energy are required to train top football athletes. Thus, such education training mode is not mature enough. With continuous practice and experience summarization, applicability of such mode can be certainly improved, and football can be supported by the society and parents.

\subsection{To improve and optimize faulty and improve coaches' level}

At present, most football coaches in primary and middle schools or PE teachers lack professional level and relevant authentication, and their educational level is mostly junior college. Usually, they only master certain basic theoretical knowledge of sports, with little practical experience. In particular, they know little about profound professional football knowledge. In the aspect of football education for primary and middle school students, relevant education and training also lack correct direction, and the foundation is obviously deficient. To better promote top football talent training ability in primary and middle schools, firstly, comprehensive quality of PE teachers and coaches should be improved. During employing relevant football education personnel, it is required to choose the personnel with strong specialized football knowledge and PE knowledge as well as teaching qualification. Moreover, there should be educational requirements for these education staffs. Only those with favorable comprehensive quality can better educate the next generation and improve comprehensive quality level of the next generation. For existing teaching staff, it is required to pay attention to professional training of PE teachers, let PE teachers participate in practice, specialized knowledge training and learning as per relevant rules, and continuously improve professional level of $\mathrm{PE}$ teachers through regular trainings. In addition, it is also required to organize PE teachers and coaches to participate in specialty exchange meeting of football, discuss advanced football skills and teaching theory mode, synthesize experience and methods of more professional personnel and improve professional ability of PE teachers and football coaches. Furthermore, some professional trainers and coaches with certain position in football field can be employed to carry out the football lectures and improve the level of PE teachers and football coaches. Football matches for middle and primary schools should be often organized and held to practice football theories so as to apply and demonstrate them. Students' physical exercise and physical quality can be enhanced while their football ability improves.

\subsection{To learn excellent football talent training mode of strong football countries}

There are many strong football countries. Germany is taken for example in this paper. This provides provide basis for football education career through studying the youth athlete training method and mode of Germany. First of all, the training system of young football athletes in Germany is highly socialized sports resources in Germany are used as the foundation, and social internal impetus is utilized to mobilize more financial resources, human resources and materials, the country and local governments carry out support, coordination and management. Social authorities and professional football sports teams formulate and execute relevant plans, and provide all-round support. Fund raising, problem management, device selection, competition organizing, athlete turnover and promotion are mostly executed by clubs or relevant schools. The system is a football sports management system with certain market proper and features. Due to different social form, the development path of football in China is also greatly different from German football education system. In China, professional football training owns rich sports funds and resources. Besides, some 
local sports systems also have idle sports resources. Local non-professional football education and training are not given sufficient attention. Thus, football talent training for primary and middle school students is limited. Therefore, China should actively learn the training and education system of strong football countries, and keep improving football talent training ability of primary and middle schools in China.

\section{Summary}

General Secretary Xi Jinping and Vice-premier Liu Yandong specified football development direction of China - to develop Chinese football to a new peak. Chinese football should not just carry forward "Shiyuki style", but also must develop football well, overall enhance youth football talent training, lay a foundation for football and drive popularization and improvement of football. The important indication of General Secretary Xi Jinping points out the direction for Chinese football development and also firms the confidence and hope of Chinese people for football. Relevant football workers have the responsibility and obligation to drive development of campus football in primary and middle schools, improve Chinese football training system and make Chinese football conform theoretical and scientific rules and advance to the correct direction through referring to the advanced football education mode of strong football countries.

\section{Acknowledgement}

This research was financially supported by “the 13th Five-year Plan” 2018 social science program of Education Department in Jilin Province, Analysis on Development Status and Countermeasures of Campus Teenager Football in Yanbian under New Situation (Grant NO.JJKH201809020SK); and also was financially supported by "the 13th Five-year Plan" 2017 research topic of Education Department in Jilin Province, Campus Football Development and Teenager Football Talent Training Mode in Yanbian under New Situation(Grant NO.GH170025).

\section{References}

[1] Guo Junhua, Status research on campus football of "national youth campus football characteristic schools” in Beijing, Capital Institute of Physical Education, 2017.

[2] Wang Man, Study on reserve talent training mode of campus football pilot school - Dongcheng District primary School in Beijing, Capital Institute of Physical Education, 2016.

[3] Hou Feng, Construction and development of diversified training mode for football Youth reserve talents in Chongqing Municipality, Chengdu Sport University, 2016.

[4] Mao Zhenming, Liu Tianbiao, On top-level design of "new campus football” - Chinese campus football from perspective of youth football athletes in Germany, Journal of Wuhan Institute of Physical Education, 2015,4906:5-11.

[5] Li Jie, Discussion on construction of Chinese youth football talent training system from perspective of successful experience of German football, China School Physical Education (Higher Education), 2017,404:48-52.

[6] He Huaxing, Study on current situation and influencing factors of campus football development in primary and middle schools of Guangzhou city, Journal of Sports Science and Technology Literature, 2016,24(11):38-40.

[7] Sun Louming, Study on sound development strategy of campus football competition in primary and middle schools, Contemporary Sports Technology, 2016,6(04):104+106.

[8] Hu Qingshan, Zeng Lijuan, Zhu Jiaxuan, Wang Jian, Thought on campus football fever - Chinese youth football reserve talent training, Journal of Beijing Sport University, 2016,39(01):126-131. 